\title{
Interdisziplinäre Kompetenzen: Modellentwicklung und diagnostische Zugänge
}

\author{
Anna M. Claus ${ }^{1}$ (D) $\cdot$ Bettina S. Wiese ${ }^{1}$ \\ Angenommen: 21. April 2021 / Online publiziert: 27. Mai 2021 \\ (c) Der/die Autor(en) 2021
}

\section{Zusammenfassung}

In diesem Beitrag in der Zeitschrift Gruppe. Interaktion. Organisation (GIO) werden die Entwicklung eines Modells interdisziplinärer Kompetenzen und zwei darauf aufbauende Messverfahren vorgestellt, nämlich ein Fragebogeninstrument für das Selbstkonzept interdisziplinärer Kompetenzen sowie ein simulationsorientierter Zugang. Interdisziplinäre Zusammenarbeit gilt als zukunftsweisend, um Innovationen an den Schnittstellen zwischen etablierten Disziplinen und Fachbereichen zu erzielen. Zugleich wird sie jedoch von den Beteiligten häufig als belastend empfunden. An dieser Stelle tragen interdisziplinäre Kompetenzen als individuelle Fähigkeiten und instrumentelle Verhaltensweisen zu erfolgreicher interdisziplinärer Zusammenarbeit bei. Das hier vorgestellte Modell interdisziplinärer Kompetenzen umfasst die Kompetenzdimensionen Initiative zum Austausch, Zielgruppenspezifische Kommunikation, Wissensintegration und Reflexion der eigenen Fachdisziplin. Die von uns konzipierte Skala zur Erfassung des Selbstkonzepts interdisziplinärer Kompetenzen kann unabhängig vom fachlichen Hintergrund der Stichprobe eingesetzt werden. Zur Reflexion des eigenen Verhaltens und als Ausgangspunkt für Entwicklungsmaßnahmen wird weiterhin ein Verhaltenssimulationsansatz vorgeschlagen, der im Vergleich jedoch einen deutlich höheren Personaleinsatz erfordert. Modell und diagnostische Zugänge ermöglichen es, im Zuge von Personalauswahl, -entwicklung und zur Evaluation der Zusammenarbeit in interdisziplinären Teams interdisziplinäre Kompetenzen von Individuen zu messen.

Schlüsselwörter Interdisziplinäre Kompetenzen · Interdisziplinäre Zusammenarbeit · Kompetenzmodell · Diagnostik

\section{Interdisciplinary competencies: model development and assessment approaches}

\begin{abstract}
This article presents the development of a model of interdisciplinary competencies and two corresponding diagnostic approaches: a scale to measure the self-concept of interdisciplinary competencies and a simulation-based approach. Interdisciplinarity promises create innovation at the borders of traditional disciplines. Interdisciplinary work, however, is often experienced as particularly stressful. Interdisciplinary competencies are defined as individual skills and instrumental behaviors that help make interdisciplinary collaborations successful. The model consists of the competency dimensions initiative for exchange, target group-specific communication, knowledge integration and reflection of the own disciplinary perspective. The proposed scale to measure the self-concept of interdisciplinary competencies is independent of individuals' disciplinary background. In addition, a behavioral simulation can be used for reflection of one's own behaviors and as a starting point for personnel development activities. This assessment approach, however, requires higher investments. The model and the corresponding diagnostic approaches allow to measure individual's interdisciplinary competencies for applications in personnel selection, personnel development, and evaluation of interdisciplinary team work.
\end{abstract}

Keywords Interdisciplinary competencies $\cdot$ Interdisciplinary collaboration $\cdot$ Competency model $\cdot$ Assessment approaches

Dr. Anna M. Claus

Anna.claus@psych.rwth-aachen.de
Institut für Psychologie, Lehrstuhl für Personal- und Organisationspsychologie, RWTH Aachen, Jägerstr. 17-19, 52056 Aachen, Deutschland 


\section{Herausforderung Interdisziplinarität}

Interdisziplinarität ist in den letzten Jahren im Wissenschaftssystem immer wichtiger geworden, aber auch in privatwirtschaftlichen Unternehmen arbeiten immer häufiger Personen mit unterschiedlichen fachlichen Hintergründen zusammen, um gemeinsam neue Produkte und Dienstleistungen zu entwickeln oder Prozesse zu optimieren. Unabhängig von der Branche ist diesen Ansätzen das innovative Potenzial gemein, das aus der Kombination verschiedener fachdisziplinärer Perspektiven entstehen soll (Sung et al. 2003). Interdisziplinarität stellt dabei einen Ansatz der fächerübergreifenden Zusammenarbeit dar, der besonders hohe Ansprüche an die Integration der disziplinären Perspektiven stellt (Repko und Szostak 2017) und grenzt sich dadurch von Multidisziplinarität, also einem Nebeneinander der Disziplinen, ab.

Interdisziplinäre Zusammenarbeit wird von vielen Erwerbstätigen als spannend und abwechslungsreich, aber zugleich auch als sehr herausfordernd beschrieben (Epstein 2005). Häufige Schwierigkeiten liegen in der Kommunikation über Fächergrenzen hinweg, dem mangelnden Verständnis für die Gegebenheiten in anderen Fachbereichen, und für die Expertise der anderen Personen. Die erlebten Schwierigkeiten können mit fachspezifischen Sozialisationsprozessen in Verbindung gebracht werden. Im Rahmen eines Studiums bzw. einer beruflichen Ausbildung entwickeln Erwerbstätige ihre berufliche Identität, die stark durch ihre jeweiligen Fachdisziplin geprägt ist (Finegold und Matousek 2007), zusätzlich entstehen organisationsspezifische Identifikationsmuster (Ashforth und Johnson 2001). Die Entwicklung von Fachkulturen mit jeweils eigenen Qualitätsstandards ermöglicht idealerweise eine unkomplizierte Zusammenarbeit innerhalb einer Disziplin, erschwert jedoch die Zusammenarbeit über Fächergrenzen hinweg (Neumeier 2007; Sung et al. 2003). Krainer und Smetschka (2014, S. 72) beschreiben die fachdisziplinäre Prägung als ,einen Rucksack an Annahmen, Haltungen und Qualitätskriterien mit dem Wissenschaftler/innen in einer je Disziplin spezifischen Sozialisation ausgestattet werden“, welcher den Akteuren nicht immer vollständig bewusst ist, aber ihr Verhalten beeinflusst. Ähnliche Sozialisationsprozesse lassen sich auch außerhalb der Wissenschaft beobachten (Antons und Piller 2015).

Zur weiteren theoretischen Erklärung der Schwierigkeiten interdisziplinären Arbeitens können Konzepte aus der Diversitätsforschung herangezogen werden. Disziplinäre Identitäten sind als Tiefenmerkmale zu verstehen, welche die Wahrnehmung und Analyse von Fragestellungen und die Entwicklung von Problemlösungen beeinflussen. Die Zusammenarbeit in disziplinär-diversen Teams birgt kreatives Potenzial, da Personen mit unterschiedlichen Erfahrungen und Herangehensweisen ihre Perspektiven zusammenbringen und das Team so über einen besonders vielfältigen Informationsstand verfügt. Gleichzeitig gehen solche Teamkonstellationen mit einem hohen Konfliktpotenzial einher, da die Perspektivenvielfalt integriert werden muss. Das Categorization-Elaboration Modell (van Knippenberg et al. 2004) verweist darauf, dass die Informationen und Perspektiven im diversen Team ausreichend exploriert werden müssen, um die Informationsvielfalt als Vorteil in der Zusammenarbeit nutzen zu können. Diese Elaboration erfordert intensive Kommunikation. Bromme et al. (2003) beschreiben die Kommunikation über Fächergrenzen hinweg als eine Form der Experten-LaienKommunikation, in der die Expertisebereiche stetig wechseln, da Personen in einem Moment Experte, im nächsten aber unerfahren sind. Um trotz dieser Wechsel zwischen eigener Expertise und Unerfahrenheit einen tiefergehenden Austausch zu ermöglichen, werden spezifische kommunikative Kompetenzen zur erfolgreichen interdisziplinären Zusammenarbeit gebraucht. In ähnlicher Weise beschreiben Steinheider et al. (2009) kognitive Wissensintegration als eine wesentliche Anforderung der interdisziplinären Zusammenarbeit. Bislang separate Wissenskorpora sollen zusammengebracht werden, um etwas Neues zu kreieren. Dazu müssen Querverbindungen zwischen den Methoden, Theorien und Wissensinhalten verschiedener Disziplinen hergestellt werden. Basierend auf einem Verständnis der anderen Perspektive kann dann eine Integration der Disziplinen angegangen werden. Diese Vorgänge sind komplex und kognitiv fordernd, aber unabdingbar, um nicht nur ein Nebeneinander (Multidisziplinarität), sondern integrative, innovative Interdisziplinarität zu erzielen.

\section{Entwicklung eines Modells interdisziplinärer Kompetenzen}

Interdisziplinäre Kompetenzen sind instrumentelle Verhaltensweisen, die zu einer erfolgreichen Bewältigung von interdisziplinären Herausforderungen beitragen (Claus und Wiese 2019). Während einzelne interdisziplinäre Herausforderungen bereits beschrieben sind (z.B. die Kommunikation über Fächergrenzen hinweg bei Bromme et al. 2003; Wissensintegration bei Steinheider et al. 2009), lag bis dato kein umfassendes und zugleich spezifisches Modell interdisziplinärer Kompetenzen vor. Ein sehr ausführliches Modell interdisziplinärer Kompetenzen wurde von Brandstädter und Sonntag (2015) vorgelegt und umfasst 40 Kompetenzdimensionen, von denen einige generisch für alle denkbaren Arbeits- und Teamsettings sind (beispielsweise Freundlichkeit und Zuverlässigkeit) und nicht spezifisch für die Herausforderungen interdisziplinärer Teams. Das neu entwickelte Modell soll hier ansetzen und zugleich umfassend, spezifisch und ökonomisch sein. Im Folgenden stellen 
wir kurz die Entwicklung des neuen Modells interdisziplinärer Kompetenzen dar; eine umfassendere Darstellung ist in Claus und Wiese (2019) zu finden.

Dem Modell liegt eine Anforderungsanalyse mit der Critical Incident Technique (CIT) zugrunde (Flanagan 1954; Koch 2010). Im ersten Schritt wurden Personen mit langjähriger Erfahrung in der interdisziplinären Zusammenarbeit in Wissenschaft und Unternehmen befragt (Expert/ inneninterviews). Die Expert/innen wurden aufgefordert, Schlüsselereignisse zu schildern, in denen Personen besonders erfolgreich interdisziplinäre Anforderungen bewältigt haben, bzw. in denen es an interdisziplinären Kompetenzen gemangelt hat. Gemeinsam mit den Expert/innen wurden erfolgskritische Verhaltensweisen abgeleitet, die zu einem positiven Outcome dieser Situationen beigetragen haben. Insgesamt wurden 38 Incidents mit 125 positiven und negativen Verhaltensweisen ausgewertet.

Ein Beispiel für einen Incident mit einem positiven Outcome wurde uns von einer Forschungsgruppenleiterin an einem interdisziplinären Forschungsinstitut geschildert, die ein positives Erlebnis mit einem Kollegen aus einem anderen Fachbereich hatte:

Am Institut gibt es begrenzte Hallenkapazität, in den Hallen sind Geräte untergebracht, auf die alle Mitarbeiter zugreifen. Da sind alle möglichen Fachrichtungen dabei. [Die Expertin] wollte in einer der Hallen Messzeiten für eine experimentelle, sozialwissenschaftliche Studie bekommen. Dafür muss die Halle ganz reserviert werden, d.h. niemand anderes kann parallel in der Halle arbeiten. Das führte zu Unmut bei den anderen Mitarbeitern. Erste Gespräche zwischen den Mitarbeitern führten zu hitzigen Diskussionen, keine Lösungsvorschläge wurden von allen eingehalten. Dann gab es ein Gespräch mit hierarchisch höherer Stufe. Dabei war ein Forschungsgruppenleiter von einem anderen Teil des Instituts dabei, der das gut gemacht hat. Er hatte viel Verständnis, hat sich die Seite [unserer Expertin] angehört, aber zugleich seine eigene vertreten. Für ihn war gesetzt, dass ein Kompromiss gefunden werden muss. Hat nicht versucht, [der Expertin] Kriterien auszureden (dass die Halle ganz reserviert werden muss). Hat das auch nicht angeprangert, sondern er hat das als Prämisse akzeptiert und dann auf Lösung hingearbeitet. Akzeptanz war zu spüren. In Formulierungen hat [die Expertin] gemerkt, dass er es nicht nachvollziehen kann, aber akzeptiert, was sie gesagt hat. Sie haben sich mündlich auf einen Kompromiss geeinigt und dann weiter gemacht. Akteur hat Kompromiss vorgeschlagen und das wurde von allen anderen akzeptiert. Das hat der Akteur herbeigeführt. Konsensorientiert, dabei nicht die eigenen Bedürfnisse herunterfahren.
Die hieraus abgeleiteten erfolgskritischen Verhaltensweisen sind ,alle Seiten anhören“, ,eigene Position vertreten“, „Prämissen Anderer akzeptieren“, „Konsensorientierung“.

In den Interviews wurden auch einige Szenen geschildert, in denen die interdisziplinäre Zusammenarbeit gescheitert ist, zum Teil mit schwerwiegenden Folgen für interdisziplinäre Forschungsprojekte und finanziellen Auswirkungen auf Unternehmen. Ein Beispiel für einen solchen Fall schilderte uns ein Manager aus der chemischen Industrie:

Ein Prozessmanager sollte die Errichtung einer neuen chemischen Anlage organisieren und planen. Dazu kommen ganz unterschiedliche Fachbereiche zusammen und bringen ihre Expertise ein. Ein Ingenieur fragt den Prozessmanager per E-Mail „Mit welcher Temperatur wird destilliert?" Es ging um eine Rohrleitung zwischen Destillationsanlage und einem Tank, das sagte er aber nicht. Die Antwort der Prozessmanagers fiel ebenfalls sehr knapp aus: „Bei $150^{\circ}$ Celsius“. Diese Kommunikation hat dazu geführt, dass die Rohrleitung komplett auf 150 Grad ausgelegt wurde. Auf $300 \mathrm{~m}$ Länge. Aber das Produkt wird eigentlich nur bei $60^{\circ} \mathrm{C}$ gefördert. Aber das war nicht die Frage. Die Frage war „bei wie viel Grad destilliert ihr?" - und darauf gab es eine Antwort. Das hat sehr hohe Mehrkosten verursacht. Ergebnis: über das Ziel hinausgeschossen, ein enormer zusätzlicher Aufwand, auch finanzieller Art, viel Frust, alle enttäuscht. Daher dann ein Debriefing gemacht und entschieden, dass in Zukunft deutlich mehr Kontextinformationen gegeben und erfragt werden müssen, wenn kommuniziert wird.

Auch aus den negativen Incidents wurden erfolgskritische Verhaltensweisen abgeleitet, die Expert/innen sollten angeben, welches Verhalten hier vermutlich zu einem besseren Ergebnis geführt hätten. Die gesammelten Incidents und Verhaltensweisen wurden anschließend in einem Workshop mit Expert/innen aus dem Bereich der Anforderungsanalyse klassifiziert. Dabei wurde bei jeder Verhaltensweise überlegt, welche zugrundeliegende Anforderung eine Person erfüllt, wenn sie diese Handlung vollzieht. Die Expert/ innen gruppierten zusammengehörige Verhaltensweisen und entwickelten Namen für die entstehenden Kategorien. Im Zuge einer solchen Anforderungsanalyse entstehen immer auch generische Anforderungsdimensionen, sodass Flanagan (1954) hier eine Überprüfung der Relevanz der gefundenen Dimensionen vorsieht. Im Rahmen des Klassifikationsworkshops wurde daher die Spezifität jeder Kompetenzdimension für interdisziplinäre Zusammenarbeit diskutiert. Aus diesem Grund wurden die Kompetenzdimensionen Kooperationsfähigkeit, Verhandlungsgeschick, Networking, Gesprächsführungskompetenz und Fachwissen aus dem Modell ausgeschlossen, da diese für jegliche 
Abb. 1 Modell interdisziplinärer Kompetenzen mit Verhaltensbeispielen

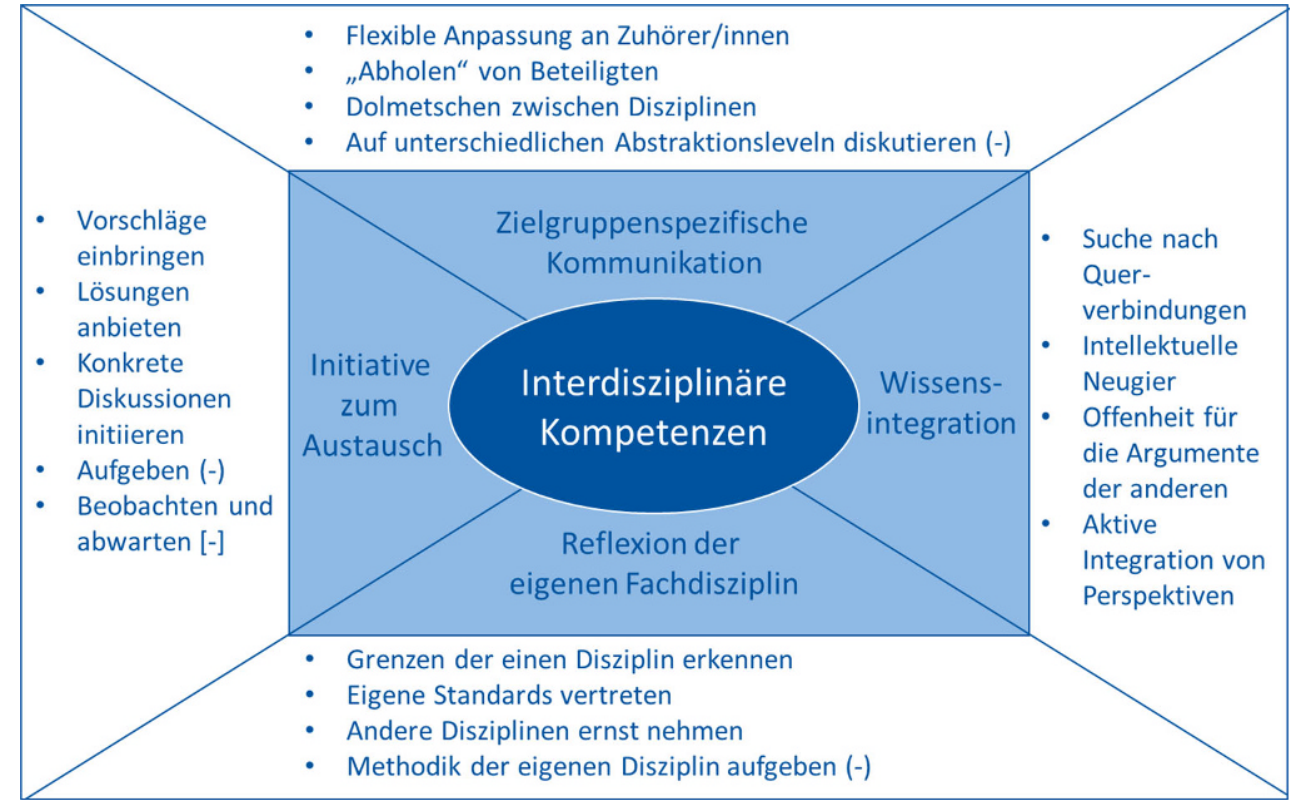

Zusammenarbeit gefordert sind und entsprechend nicht spezifisch für interdisziplinäre Anforderungen sind.

Das so entwickelte Modell interdisziplinärer Kompetenzen besteht aus vier Kompetenzdimensionen: Initiative zum Austausch, Zielgruppenspezifische Kommunikation, Wissensintegration und Reflexion der eigenen Fachdisziplin (siehe Abb. 1). Initiative zum Austausch wird als proaktive Kompetenz definiert, interdisziplinäre Diskussionen herbeizuführen. Relevante Verhaltensweisen sind Vorschläge einbringen, konkrete Diskussionen initiieren, nicht aufgeben und nicht darauf warten, dass andere aktiv werden. Zielgruppenspezifische Kommunikation bezieht sich auf die Fähigkeit, die eigene Kommunikation flexibel an die jeweilige Zuhörerschaft anzupassen. Dazu gehört es, zwischen verschiedenen Disziplinen zu ,übersetzen“, geduldig zu erklären, Fachbegriffe behutsam einzuführen und auf Missverständnisse hinzuweisen. Wissensintegration bezieht sich auf die kognitive Kompetenz, Verbindungen zwischen verschiedenen Wissensbereichen herzustellen. Wir verstehen darunter eine intellektuelle Neugierde, Offenheit für andere Herangehensweisen und die aktive Suche nach Querverbindungen zwischen bislang separaten Themenfeldern. Reflexion der eigenen Fachdisziplin umfasst die distanzierte Rückschau auf die eigene disziplinäre Sozialisation. Dazu gehört, die Möglichkeiten aber auch Grenzen der eigenen Fachdisziplin $\mathrm{zu}$ erkennen. Zu dieser Kompetenzdimension zählt beispielsweise, die eigenen Qualitätskriterien begründen und aufrechterhalten zu können.

Das Zusammenspiel dieser vier Kompetenzdimensionen ermöglicht es Individuen, erfolgreich interdisziplinäre Arbeitsherausforderungen zu bewältigen. Die Entwicklung dieser Kompetenzen kann sowohl on the job erfolgen, d.h. in der Auseinandersetzung mit interdisziplinären Arbeitssituationen, als auch gefördert werden durch spezifische Interventionen, wie sie zum Beispiel für die Wissensintegration bereits existieren (Bertsche und Bullinger 2007).

\section{Wege der Erfassung von interdisziplinären Kompetenzen}

Nachfolgend stellen wir zwei Wege der Erfassung der modellbasierten Kompetenzdimensionen vor: Ein Selbstberichtsinstrument und einen simulationsbasierten Ansatz.

\subsection{Selbstberichtsinstrument}

Bei der hier beschriebenen Entwicklung des Instruments zur Erfassung interdisziplinärer Kompetenzen wurden verschiedene Zielsetzungen verfolgt: Das neue Instrument sollte das Modell interdisziplinärer Kompetenzen abbilden, ökonomisch einzusetzen sein und als Selbstbericht auf das Selbstkonzept der Kompetenzen abzielen. Ein Selbstbericht bildet dabei das Selbstkonzept der Befragten in Bezug auf ihre interdisziplinären Kompetenzen ab, d.h. ihre kognitiven Fähigkeitsrepräsentationen und Einschätzungen (vgl. Sonntag und Schäfer-Rauser 1993). Selbstkonzept und Verhalten stehen dabei in einem reziproken Zusammenhang: Das eigene Verhalten in interdisziplinären Settings prägt das Selbstkonzept interdisziplinärer Kompetenzen, aber das Selbstkonzept beeinflusst zugleich mit welcher Einstellung, Motivation und welchem Verhalten eine Person sich in eine interdisziplinäre Arbeitssituation begibt. Eine ausführliche 
Darstellung der Skalenentwicklung findet sich bei Claus und Wiese (2019).

\subsubsection{Itementwicklung}

Der Kompetenzgedanke wurde bei der Itementwicklung beispielsweise durch Formulierungen wie „Mir fällt es leicht ..." oder „Ich kann gut“ als Satzeinleitungen von der Erstautorin umgesetzt. Inhaltlich greifen die Items die Verhaltensweisen aus der Anforderungsanalyse in den jeweiligen Kompetenzdimensionen auf. Die so entstandenen Items wurden von vier Wissenschaftlerinnen auf ihre Augenscheinvalidität für die jeweilige Dimension überprüft und bei Bedarf überarbeitet oder ausgeschlossen. Im ersten Schritt entstanden so 66 Items zur Messung der vier Kompetenzdimensionen, von denen später empirisch die geeignetsten ausgewählt wurden, um eine ökonomische und valide Skala zu entwickeln.

\subsubsection{Gütekriterien}

In zwei Studien wurden die Gütekriterien, insbesondere die Reliabilität und die Validität des entwickelten Instruments untersucht. Für Studie 1 wurden dazu 315 Erwerbstätige, die zum Zeitpunkt der Untersuchung interdisziplinär arbeiteten, befragt. Teilnehmende sollten mindestens einmal pro Woche inhaltlich mit einer Person aus einer anderen Fachdisziplin zusammenarbeiten, sodass hier eine recht offene Definition von Interdisziplinarität gewählt wurde. Die Studienteilnehmenden (55,3\% Frauen) waren überwiegend Hochschulabsolvent/innen $(98,4 \%)$ und arbeiteten hauptsächlich in der Wissenschaft $(84,5 \%)$. Eine Teilstichprobe von 202 Personen nahm an einer Messwiederholung nach etwa 14 Tagen teil.

Zunächst wurden anhand deskriptiver Itemcharakteristiken jene Items ausgeschlossen, die zu wenig Varianz aufwiesen bzw. nur eine sehr geringe Itemschwierigkeit hatten. Diese Items waren nicht in der Lage, zwischen Personen mit hohen und niedrigen Merkmalsausprägungen zu differenzieren. Im nächsten Schritt wurden durch faktorenanalytische Verfahren (zunächst exploratorische Faktorenanalysen, später exploratorische Strukturgleichungsmodelle bzw. konfirmatorische Faktorenanalysen) Items mit uneindeutigen Ladungsmustern und sehr hohen Kreuzladungen ausgeschlossen. In diesem iterativen Prozess wurden sowohl die empirischen Analyseergebnisse als auch die inhaltliche Ausgestaltung der Modelldimensionen berücksichtigt. Das Ergebnis sind 13 Items, welche die vier Modelldimensionen abdecken (siehe Tab. 1).

Eine zusätzlich eingesetzte exploratorische Strukturgleichungsmodellierung (ESEM; Asparouhov und Muthén 2009) spiegelte die mittels CIT entwickelte Modellstruktur mit korrelierten Kompetenzdimensionen wider. Die entwickelten Items laden hauptsächlich auf einen latenten Kompetenzfaktor, teilweise bestehen daneben geringe Kreuzladungen. Der Modellfit für das Modell mit korrelierten Dimensionen ist gut $\left(\chi^{2}=39,82 ; \mathrm{df}=32 ; \mathrm{CFI}=0,992\right.$; $\mathrm{TLI}=0,981$; RMSEA $=0,028$; SRMR $=0,021$ ). Details finden sich in Claus und Wiese (2019). Einzig in der Dimension Reflexion der eigenen Fachdisziplin ergab sich eine Änderung: diese enthielt ursprünglich auch Items, welche die Wertschätzung für andere Fachdisziplinen ausdrücken. Diese Items werden zur klaren Abgrenzung von motiva-

Tab. 1 Items zur Messung interdisziplinärer Kompetenzen im Selbstbericht

Initiative zum Austausch $(\alpha=0,68)$

Es fällt mir leicht, konkrete Vorschläge zu machen um eine Diskussionsgrundlage für die interdisziplinäre Arbeitsgruppe zu schaffen.

Ich bin häufig die Person, von der Ideen für interdisziplinäre Projekte ausgehen.

Es fällt mir leicht, bei einer fächerübergreifenden Besprechung die Initiative zu ergreifen.

Zielgruppenspezifische Kommunikation ( $\alpha=0,67)$

Wenn ich interdisziplinär arbeite, fällt es mir schwer, darauf zu achten, keine unnötigen Fachbegriffe zu verwenden. (umpolen)

In interdisziplinären Arbeitsgruppen fällt es mir schwer, meine Sichtweise verständlich zu machen. (umpolen)

Für mich ist es kein Problem, meine Ausdrucksweise in interdisziplinären Arbeitsgruppen so anzupassen, dass mich alle verstehen können.

Es fällt mir schwer, mich sprachlich auf fachfremde Mitglieder in der Arbeitsgruppe einzulassen. (umpolen)

Wissensintegration $(\alpha=0,73)$

Wenn ich interdisziplinär arbeite, kann ich gut Wissen aus verschiedenen Fachbereichen verbinden und integrieren.

In interdisziplinären Arbeitsgruppen gelingt es mir, verschiedene Fachgebiete inhaltlich zu verknüpfen.

In interdisziplinären Arbeitsgruppen kann ich gut durchdringen, womit andere Mitglieder sich inhaltlich beschäftigen.

Reflexion der eigenen Fachdisziplin $(\alpha=0,72)$

Ich halte auch in interdisziplinären Arbeitsgruppen die Qualitätskriterien meiner eigenen Fachdisziplin hoch.

Ich kann sehr genau benennen, für welche Fragestellungen meine Disziplin idealerweise „zuständig“ ist und worin sie sich von benachbarten Disziplinen unterscheidet.

Ich kann sehr genau benennen, worin die methodischen und inhaltlichen Besonderheiten meiner Disziplin bestehen.

6-stufige Antwortskala von „,trifft nicht zu“ bis ,trifft voll zu“ 
tionalen Aspekten der Zusammenarbeit nicht als Teil der Reflexion der Fachdisziplin geführt.

In Bezug auf die Reliabilität wurden Messinvarianz, interne Konsistenz und Retest-Reliabilität über das 14-tägige Retest-Intervall bestimmt. Es zeigte sich eine starke, aber keine strikte Messinvarianz über die Zeit. Daraus lässt sich schließen, dass Faktorladungen, Varianz-Kovarianz-Strukturen der Faktoren und Achsenschnittpunkte (Intercepts) zwischen beiden Zeitpunkten vergleichbar sind. Zudem zeigte sich kurzfristige Stabilität durch Faktorkorrelationen von $0,70-0,92$ (vgl. Claus und Wiese 2019). Für manifest gebildete Skalenmittelwerte ergaben sich hinreichende interne Konsistenzen der Skalen (vgl. Tab. 1).

Die Validität von Modell und Instrument wurde in einer zweiten Studie beleuchtet (vgl. Claus und Wiese 2019). Hierfür wurden $N=448$ Erwerbstätige als Zielpersonen rekrutiert $(57,6 \%$ Frauen; durchschnittlich 42 Jahre alt, $28,8 \%$ arbeiteten im Wissenschaftssystem). Erneut wurden Personen angesprochen, die regelmäßig interdisziplinär arbeiten. Dazu wurden Organisationen kontaktiert, die vermehrt interdisziplinäre Zusammenarbeit erfordern (beispielsweise Sozialpädiatrische Zentren, Unternehmen mit dem Schwerpunkt Nachhaltigkeit, etc.). Etwa ein Drittel von ihnen $(n=153)$ konnten eine Kollegin oder einen Kollegen aus einem anderen Fachbereich für eine Fremdeinschätzung gewinnen.

In dieser Studie wurden konvergente und prädiktive Validität untersucht. Es zeigten sich mittlere positive $\mathrm{Zu}$ sammenhänge zwischen den selbstberichteten interdisziplinären Kompetenzen auf der einen Seite und Interesse an interdisziplinärer Zusammenarbeit, Präferenz für Teamarbeit im Allgemeinen und Selbstwirksamkeitsüberzeugungen für Teamarbeit auf der anderen Seite. Zudem konnten wir einen positiven Zusammenhang zwischen Unterstützung der Vorgesetzten für Interdisziplinarität und selbstberichteter Zielgruppenspezifischer Kommunikation ( $r=0,23$; $p<0,001)$ und Wissensintegration $(r=0,19 ; p<0,01)$ feststellen. Personen, die über eine höhere Abhängigkeit von interdisziplinären Kolleginnen und Kollegen berichteten, um ihre eigene Arbeitstätigkeit auszuführen, berichteten zudem höhere interdisziplinäre Kompetenzen. So lässt sich zusammenfassen, dass sowohl Einstellungen zur interdisziplinären Zusammenarbeit als auch organisationale Merkmale (Unterstützung durch Vorgesetzte, Interdependenz) mit selbstberichteten interdisziplinären Kompetenzen zusammenhängen (konvergente Validität).

Die interdisziplinären Kolleginnen und Kollegen gaben Auskunft über die Effektivität der interdisziplinären Zusammenarbeit im Team und ihre Zufriedenheit mit dem interdisziplinären Team. In den Analysen zur prädiktiven Validität wurde geprüft, ob interdisziplinäre Kompetenzen über allgemeine Teamkompetenzen (Halfhill und Nielsen 2007) hinaus diese interdisziplinären Outcomes vorhersagen kön- nen. Allgemeine Teamkompetenzen sagten alleine genommen Effektivität $\left(R^{2}=0,14 ; p<0,001\right)$ und Zufriedenheit $\left(R^{2}=0,07 ; p<0,01\right)$ im Selbstbericht, nicht aber im Fremdbericht vorher $\left(R_{\text {Effektivitatt }}=0,07 ; R^{2}\right.$ Zufriedenheit $=0,08$; beides n.s.). Interdisziplinäre Kompetenzen hingegen vermochten über allgemeine Teamkompetenzen hinaus Effektivität $\left(\right.$ Gesamt $-R^{2}$ Selbstbericht $=0,21 ; p<0,001$; Gesamt $-R^{2}$ Fremdbericht $=0,14 ; p<0,05)$ und Zufriedenheit (Gesamt $-R^{2}$ Selbstbericht $=0,17 ; p<0,001$; Gesamt $-R^{2}$ Fremdbericht $\left.=0,12 ; p<0,05\right)$ vorherzusagen. Diese Analysen basieren allerdings ausschließlich auf querschnittlichen Analysen.

\subsection{Verhaltenssimulation}

Interdisziplinäre Kompetenzen haben eine starke interaktive Verhaltenskomponente - der effektive Umgang mit herausfordernden interdisziplinären Situationen zeigt sich im direkten Austausch mit einer Person aus einer anderen Fachdisziplin. Daher ist trotz des erheblichen Aufwands in der Konzeption und durch den Einsatz von Rollenspielern und die erforderlichen Mehrfachbeobachtungen eine Verhaltensmessung eine bedenkenswerte Option. Es bieten sich die Erfassung in Verhaltenssimulationen wie Rollenspielen und Vortragsübungen, die häufig in Assessment Centern eingesetzt werden, oder Situational Judgement Tests an. Dabei werden üblicherweise Szenarien entwickelt, in denen eine Situation hinreichend genau geschildert wird, damit die Teilnehmenden sich gut hineinversetzen können. Verhaltensmessungen mit interdisziplinären Interaktionen können dabei nicht unabhängig von der Fachdisziplin der Teilnehmenden geplant werden. Wir berichten hier von einem Orientierungscenter für Nachwuchswissenschaftler/-innen aus dem MINT-Bereich, in dem interdisziplinäre Kompetenzen in drei Aufgaben gemessen wurden (Claus et al. 2020). Das Orientierungscenter war Teil eines umfangreichen Forschungsprojektes (Burk et al. 2014; Burk und Wiese 2018) zu den Karriereentscheidungen und Karrierewegen von Nachwuchswissenschaftler/-innen.

Für das Orientierungscenter wurde ein Szenario entwickelt, in das die drei Aufgaben zur Messung der interdisziplinären Kompetenzen eingebettet waren. Die Entwicklung der Aufgaben erfolgte in Anlehnung an die Trait-Activation-Theory (Lievens und Schollaert 2011) und unter Rückbezug auf die Critical Incidents aus den Experteninterviews. Die Trait-Activation-Theory (Lievens und Schollaert 2011) postuliert, dass dauerhafte Personencharakteristika (,traits") situationsspezifisch aktiviert werden. Um also bestimmte Kompetenzen in einer Situation beobachten zu können, muss die Situation so gestaltet werden, dass sie Personen dazu animiert, ihre Kompetenzen zu zeigen.

Das Szenario: An der Universität gibt es eine interne Ausschreibung zur Förderung interdisziplinärer Projekte. Diese Projekte sollen sich entweder in das Themenfeld 
„Trinkwasserknappheit“ oder in das Themenfeld „Elektromobilität" einbetten lassen. Diese Themen wurden mit der Zielgruppe MINT-Wissenschaftler/innen im Blick gewählt, in der Annahme, dass möglichst viele Teilnehmenden einen Bezug zu einem dieser Felder herstellen können.

Aufgabe 1 des Orientierungscenters ist die Teilnahme an einem interdisziplinären Kolloquium, das im Rahmen der Ausschreibung zur Projektförderung stattfindet. Die Teilnehmenden sollen einen Vortrag vor einem interdisziplinären Publikum halten und dabei ihre aktuelle Forschung vorstellen und die Bezüge zum gewählten Themenfeld (Trinkwasserknappheit oder Elektromobilität) aufzeigen. Zudem sollen sie erläutern, welche Kompetenzen sie selbst bzw. ihre Disziplin für ein mögliches Projekt mitbringen und wofür sie auf andere Fachdisziplinen angewiesen sein würden. In dieser Aufgabe werden die Zielgruppenspezifische Kommunikation der Teilnehmenden und ihre Reflexion der eigenen Fachdisziplin erfasst.

In Aufgabe 2 des Orientierungscenters treffen die Teilnehmenden auf eine potenzielle Kooperationspartnerin. Den Teilnehmenden wird ein Video mit dem Kolloquiumsbeitrag dieser Kooperationspartnerin gezeigt, einer Politikwissenschaftlerin, mit der er/sie auf Wunsch der Vorgesetzten einen gemeinsamen Antrag für die interne Projektförderung vorbereiten soll. Sie arbeitet an einer ganz anderen Fragestellung, die sich nur indirekt auf die ausgeschriebenen Themenfelder anwenden lässt. Im Gespräch sollen die Teilnehmenden eine konkrete Projektidee entwickeln. Die Kooperationspartnerin wird dabei von einer professionellen Schauspielerin verkörpert, die für verschiedene Einwände und Vorschläge der Teilnehmenden standardisierte Reaktionen spielt (entsprechend der TraitActivation-Theory). Dies sichert eine hohe Vergleichbarkeit zwischen verschiedenen Teilnehmenden, auch wenn Rollenspiele grundsätzlich nur bedingt standardisiert werden können. In dieser Aufgabe sollen alle vier interdisziplinären Kompetenzen Initiative zum Austausch, Zielgruppenspezifische Kommunikation, Wissensintegration und Reflexion der eigenen Fachdisziplin beobachtet werden.

Aufgabe 3 ist ein biographisches Interview. In diesem werden die Teilnehmenden angeleitet, ihr eigenes, vergangenes Verhalten in interdisziplinären Situationen zu schildern und zu reflektieren. Die Fragen zielen dabei auf Situationen ab, in denen die Teilnehmenden Initiative zum Austausch, Zielgruppenspezifische Kommunikation oder Wissensintegration gezeigt haben.

An dem Orientierungscenter haben 127 Nachwuchswissenschaftler/innen aus dem MINT-Bereich teilgenommen. Das Verhalten wurde auf Video aufgezeichnet und später von jeweils 3 Beobachter/innen aus einem Pool von 11 Beobachtenden auf standardisierten Instrumenten bewertet. Die Instrumente zielten auf die Erfassung von relevanten Verhaltensweisen für die jeweiligen Kompetenzdimen- sionen ab. Die Beobachtenden wurden bezüglich Verhaltensbeobachtungen im Allgemeinen (z.B. Beobachtungsfehler) und den Kompetenzen und Beobachtungsinstrumenten im Speziellen (Bezugsrahmentraining) geschult. Insgesamt wurde die Leistung in der Präsentationsaufgabe besser bewertet als in der Dialog- und Interviewaufgabe (vgl. Claus et al. 2020). Mit Blick auf die einzelnen Kompetenzdimensionen deutete sich an, dass es den Nachwuchswissenschaftler/innen in dem vorgegebenen Setting leichtfiel, proaktiv aufzutreten und Initiative zum Austausch zu zeigen. Die Integration von unterschiedlichen Perspektiven und Wissensbeständen im Dialog war insgesamt betrachtet am schwierigsten und wurde im Schnitt schlechter bewertet.

Die Ergebnisse machen deutlich, dass es tatsächlich sinnvoll ist, das Szenario an den jeweiligen fachlichen Hintergrund anzupassen. Unsere Teilnehmenden konnten zwischen zwei Themenfeldern für das hypothetische Projekt wählen - Trinkwasserknappheit oder Elektromobilität. Während sich in der Bewertung der Leistung der Teilnehmenden kein bedeutsamer Unterschied zwischen beiden Szenarien zeigt, gab es einen signifikanten Effekt der fachlichen Nähe der Teilnehmenden zum Szenario auf die Bewertung ihrer Kompetenzen. Teilnehmer/innen, die selbst angaben, gut vertraut mit dem jeweiligen Themenfeld zu sein, wurde mehr Initiative zum Austausch, mehr Wissensintegrationskompetenz und mehr Reflexion der eigenen Fachdisziplin diagnostiziert. Bei zukünftigen Einsätzen sind demnach Szenarien zu wählen, die es den Teilnehmenden ermöglichen, ausgehend von ihrem Vorwissen ihr volles Potenzial interdisziplinärer Kompetenzen zu zeigen.

\subsection{Vergleich der diagnostischen Zugänge}

Das Selbstberichtsinstrument bietet eine ökonomische Möglichkeit, das Selbstkonzept interdisziplinärer Kompetenzen zu erfassen. Dabei ist es unabhängig von der Fachdisziplin der Teilnehmenden einsetzbar und muss nicht auf die Vorkenntnisse der Stichprobe adaptiert werden. Da die Teilnehmenden ihre individuellen Einschätzungen zu ihrem interdisziplinären Verhalten angeben sollen, erfordert dieses Instrument erste Erfahrungen mit fachübergreifender Zusammenarbeit von den Teilnehmenden und Selbstreflexionsfähigkeit.

Im Gegensatz dazu bringt die Simulationsübung die Messung interdisziplinärer Kompetenzen auf die Verhaltensebene. Dies ermöglicht es auch Teilnehmenden, die noch keine Erfahrungen mit der fachübergreifenden $\mathrm{Zu}$ sammenarbeit haben, sich auszuprobieren. Weiterhin kann dieser Zugang gut im Rahmen von Personalentwicklungsmaßnahmen eingesetzt werden, sowohl zur Identifikation von Entwicklungsbereichen als auch zur Evaluation von Interventionen. Verhaltenssimulationen sind weniger anfällig 
für Verfälschungen im Sinne der sozialen Erwünschtheit (Dürr und Klehe 2018). Ein solcher simulationsorientierter Zugang ist jedoch ressourcenintensiv, da zum einen die Simulation an den jeweiligen Kontext angepasst werden muss, aber auch die Durchführung und Beobachtung der Verhaltenssimulation den Einsatz geschulten Personals erfordern. Für eine realistische Situationsbeschreibung sind Detailbeschreibungen wichtig, die den Teilnehmenden ein Sich Hineinversetzen in die Situation ermöglichen. Auf eine solche Einordnung zu verzichten oder Aufgaben explizit in einen ,fremden“ Fachbereich zu verlegen ist zu vermeiden, da die disziplinäre Verortung als Ausgangspunkt eine Voraussetzung für interdisziplinäres Handeln darstellt.

\section{Diskussion}

Interdisziplinäre Zusammenarbeit kann ein zweischneidiges Schwert sein: die Perspektivenvielfalt kann große Innovationen ermöglichen, zugleich erschwert sie die $\mathrm{Zu}-$ sammenarbeit und kann Projekte scheitern lassen. In diesem Artikel standen Kompetenzen von Erwerbstätigen im Mittelpunkt, die interdisziplinäre Zusammenarbeit gelingen lassen. Wir stellen ein kompaktes und spezifisches Modell interdisziplinärer Kompetenzen mit vier Kompetenzdimensionen vor: Initiative zum Austausch, Zielgruppenspezifische Kommunikation, Wissensintegration und Reflexion der eigenen fachdisziplinären Perspektive. Dieses qualitativ-induktiv entwickelte Kompetenzmodell weist erwartungsgemäß Bezüge zu interdisziplinären Herausforderungen auf, die bereits durch andere Autor/innen beschrieben wurden (z.B. die Kommunikation über Fächergrenzen hinweg bei Bromme et al. 2003; oder Wissensintegration bei Steinheider et al. 2009). Der Vorteil dieses Kompetenzmodells liegt in der kompakten und zugleich umfassenden Beschreibung relevanter Kompetenzen im Kontext interdisziplinären Handelns. Die empirische Modellentwicklung stellt sicher, dass sich die Kompetenzdimensionen im beruflichen Handeln als erfolgskritische Unterscheidungsmerkmale bewiesen haben und daher in der Praxis verortet sind. Das Kompetenzmodell kann in verschiedenen Kontexten Anwendung finden: Beispielsweise als Grundlage für einen Kick-Off-Prozess in neu zusammengetretenen interdisziplinären Teams oder als Ausgangspunkt für gezielte Interventionen zur Verbesserung der interdisziplinären Zusammenarbeit.

Basierend auf dem Kompetenzmodell wurde ein Selbstberichtsinstrument sowie ein verhaltenssimulierender $\mathrm{Zu}$ gang entwickelt, welche interdisziplinäre Kompetenzen erfassen. Das Fragebogeninstrument ist ökonomisch einsetzbar und wurde in zwei Studien erprobt. Hier zeigte sich eine zufriedenstellende Güte in Bezug auf die Reliabilität und die Validität des Instruments. Das Fragebogeninstrument kann als Ansatzpunkt zur Reflexion des eigenen Verhaltens in interdisziplinären Teams genutzt werden, aber auch als Screening-Tool für Personalentwicklungsmaßnahmen. Weiterhin sehen wir Anwendungsmöglichkeiten in der Evaluation von Interventionen. Zudem beschreiben wir die Möglichkeit, Verhaltensdaten zu den interdisziplinären Kompetenzen zu erfassen. Derartige Simulationen erfordern eine genaue Anpassung auf die jeweilige Stichprobe und sind dadurch eingeschränkter in ihren Einsatzmöglichkeiten. Die Digitalisierung eröffnet hier perspektivisch neue Möglichkeiten, die Erfassung ökonomischer zu gestalten durch eine ortsunabhängige Durchführung der Simulation (vgl. Langer et al. 2017). Verhaltensdaten zu interdisziplinären Kompetenzen können in Personalauswahlprozessen eingesetzt werden, um Positionen mit interdisziplinärem Profil zu besetzen.

\section{Fazit}

Interdisziplinäre Zusammenarbeit wird häufig als belastend erlebt - das Gefühl nicht richtig verstanden zu werden oder nicht ganz zu verstehen, warum andere in einer bestimmten Weise vorgehen, beeinträchtigen das Miteinander. Das hier entwickelte Kompetenzmodell soll als Ausgangspunkt dazu dienen, interdisziplinäre Zusammenarbeit zu evaluieren und zu verbessern. Die Skala zur Erfassung des Selbstkonzepts interdisziplinärer Kompetenzen sowie simulationsbasierte Verhaltensaufgaben können genutzt werden, um das eigene Verhalten in interdisziplinären Settings zu reflektieren und Entwicklungsbedarfe zu ermitteln. Auch eine Weiterentwicklung zum Zwecke der Personalauswahl ist denkbar. Das Modell interdisziplinärer Kompetenzen zeigt auf, wie interdisziplinäre Zusammenarbeit auf individueller Ebene erfolgreich gestaltet werden kann, sodass interdisziplinäre Teams ihr Potenzial entfalten können.

Danksagung Die Autorinnen bedanken sich für finanzielle Unterstützung beim Bundesministerium für Bildung und Forschung (Förderkennziffern 16FWN009).

Funding Open Access funding enabled and organized by Projekt DEAL.

Open Access Dieser Artikel wird unter der Creative Commons Namensnennung 4.0 International Lizenz veröffentlicht, welche die Nutzung, Vervielfältigung, Bearbeitung, Verbreitung und Wiedergabe in jeglichem Medium und Format erlaubt, sofern Sie den/die ursprünglichen Autor(en) und die Quelle ordnungsgemäß nennen, einen Link zur Creative Commons Lizenz beifügen und angeben, ob Änderungen vorgenommen wurden.

Die in diesem Artikel enthaltenen Bilder und sonstiges Drittmaterial unterliegen ebenfalls der genannten Creative Commons Lizenz, sofern sich aus der Abbildungslegende nichts anderes ergibt. Sofern das betreffende Material nicht unter der genannten Creative Commons Lizenz steht und die betreffende Handlung nicht nach gesetzlichen Vorschriften erlaubt ist, ist für die oben aufgeführten Weiterverwendungen des Materials die Einwilligung des jeweiligen Rechteinhabers einzuholen. 
Weitere Details zur Lizenz entnehmen Sie bitte der Lizenzinformation auf http://creativecommons.org/licenses/by/4.0/deed.de.

\section{Literatur}

Antons, D., \& Piller, F. T. (2015). Opening the black box of "not invented here": attitudes, decision biases, and behavioral consequences. Academy of Management Perspectives, 29(2), 193-217. https:// doi.org/10.5465/amp.2013.0091.

Ashforth, B.E., \& Johnson, S. A. (2001). Which hat to wear? The relative salience of multiple identities in organizational contexts. In M. A. Hogg \& D. J. Terry (Hrsg.), Social identity processes in organisational contexts (S. 85-96). Philadelphia, PA: Psychology Press.

Asparouhov, T., \& Muthén, B. (2009). Exploratory structural equation modeling. Structural Equation Modeling, 16, 397-438. https:// doi.org/10.1080/10705510903008204.

Bertsche, B., \& Bullinger, H.-J. (Hrsg.). (2007). Entwicklung und Erprobung innovativer Produkte - Rapid Prototyping. Grundlagen, Rahmenbedingungen und Realisierung. Berlin Heidelberg: Springer.

Brandstädter, S., \& Sonntag, K. (2015). Interdisziplinäre Zusammenarbeit - wie kann der Dialog über die Fachgrenze hinweg optimiert werden? In VerANTWORTUNG für die Arbeit der Zukunft. Bericht zum 61. Frühjahrskongress der Gesellschaft für Arbeitswissenschaften. Dortmund: GfA Press.

Bromme, R., Jucks, R., \& Rambow, R. (2003). Wissenskommunikation über Fächergrenzen: Ein Trainingsprogramm. Wirtschaftspsychologie, 3, 94-102.

Burk, C.L., \& Wiese, B. S. (2018). Nah der Wissenschaft und fern der Führung in einer Fach-/Expertenlaufbahn? Industrielle Karrierepfade Promovierter aus den MINT-Fächern. Gruppe. Interaktion. Organisation: Zeitschrift für Angewandte Organisationspsychologie, 49, 23-33.

Burk, C.L., Alisic, A., Stertz, A., Teeuwen, S., \& Wiese, B. S. (2014). Überfachliche Anforderungsprofile von Natur- und Ingenieurwissenschaftlern in Wissenschaft und Industrie. 49. Kongress der Deutschen Gesellschaft für Psychologie, Bochum.

Claus, A. M., \& Wiese, B.S. (2019). Development and test of a model of interdisciplinary competencies. European Journal of Work and Organizational Psychology, 28, 191-205. https://doi.org/10.1080/ 1359432X.2019.1567491.

Claus, A.M., Arend, M.G., Burk, C.L., Kiefer, C., \& Wiese, B.S. (2020). Cross-classified models in I/O psychology. Journal of Vocational Behavior, 120, 103447. https://doi.org/10.1016/j.jvb. 2020.103447

Dürr, D., \& Klehe, U.-C. (2018). Using the theory of planned behavior to predict faking in selection exercises varying in fidelity. Journal of Personnel Psychology, 17, 155-160. https://doi.org/10.1027/ 1866-5888/a000211.

Epstein, S.L. (2005). Making interdisciplinary collaboration work. In S.J. Derry, C.D. Schunn \& M. A. Gernsbacher (Hrsg.), Interdisciplinary collaboration. An emerging cognitive science (S. 245-263). New York, NY: Lawrence Erlbaum.

Finegold, D., \& Matousek, R. (2007). US efforts to create a new professional identity for the bioscience industry. In A. Brown, S. Kirpal \& F. Rauner (Hrsg.), Identities at work. Technical and vocational education and training: issues, concerns and prospects (S. 361-390). Berlin Heidelberg: Springer. https://doi.org/ 10.1007/978-1-4020-4989-7.

Flanagan, J.C. (1954). The critical incident technique. Psychological Bulletin, 51, 327-359. https://doi.org/10.1037/h0061470.

Halfhill, T. R., \& Nielsen, T.M. (2007). Quantifying the "softer side" of management education: an example using teamwork competencies. Journal of Management Education, 31(1), 64-80. https:// doi.org/10.1177/1052562906287777. van Knippenberg, D., De Dreu, C. K. W., \& Homan, A. C. (2004). Work group diversity and group performance: an integrative model and research agenda. Journal of Applied Psychology, 89, 1008-1022. https://doi.org/10.1037/0021-9010.89.6.1008.

Koch, A. (2010). Die Task-Analysis-Tools (TAToo) - Entwicklung, empirische und praktische Prüfungen eines Instrumentes für Anforderungsanalysen. Dresden: Technische Universität Dresden.

Krainer, L., \& Smetschka, B. (2014). Ein Forschungsteam finden. In G. Dressel, W. Berger, K. Heimerl \& V. Winiwarter (Hrsg.), Interdisziplinär und transdisziplinär forschen (S. 65-78). Bielefeld: transcript.

Langer, M., König, C. J., \& Krause, K. (2017). Examining digital interviews for personnel selection: applicant reactions and interviewer ratings. International Journal of Selection and Assessment, 25, 371-382. https://doi.org/10.1111/ijsa.12191.

Lievens, F., \& Schollaert, E. (2011). Adjusting exercise design in assessment centers: theory, practice, and research. In N. Povah \& G. C. Thornton (Hrsg.), Assessment centres and global talent management (S. 47-60). Farnham: Gower.

Neumeier, R. (2007). Interdisziplinäres Forschen. Frankfurt am Main: Peter Lang.

Repko, A. F., \& Szostak, R. (2017). Interdisciplinary research: process and theory. Hoboken: SAGE.

Sonntag, K., \& Schäfer-Rauser, U. (1993). Selbsteinschätzung beruflicher Kompetenzen bei der Evaluation von Bildungsmaßnahmen. Zeitschrift für Arbeits- und Organisationspsychologie, 37, 163-171.

Steinheider, B., Bayerl, P.S., Menold, N., \& Bromme, R. (2009). Entwicklung und Validierung einer Skala zur Erfassung von Wissensintegrationsproblemen in interdisziplinären Projektteams (WIP). Zeitschrift für Arbeits- und Organisationspsychologie, 53, 121-130. https://doi.org/10.1026/0932-4089.53.3.121.

Sung, N. S., Gordon, J. I., Rose, G. D., Getzoff, E. D., Kron, S. J., Mumford, D., Onuchic, J.N., Scherer, N.F., Sumners, D.L., \& Kopell, N. J. (2003). Educating future scientists. Science, 301, 1485. https://doi.org/10.1126/science.10866133.

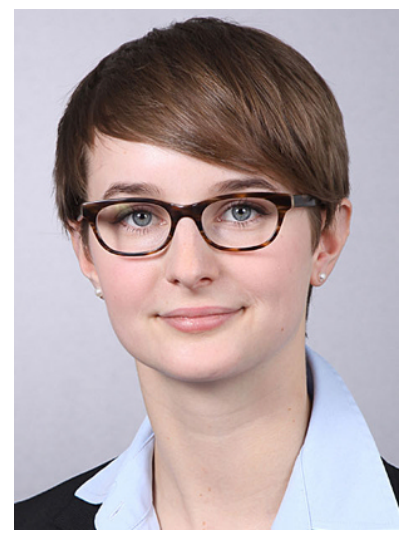

Dr. Anna M. Claus (B.Sc., M.Sc. und Promotion in Psychologie an der RWTH Aachen) ist Postdoktorandin am Lehrstuhl für Personalund Organisationspsychologie der RWTH Aachen University. In ihrer Forschung untersucht sie verschiedene Aspekte von Interdisziplinarität im Arbeits- und Studienkontext und interessiert sich für methodische Innovationen. 


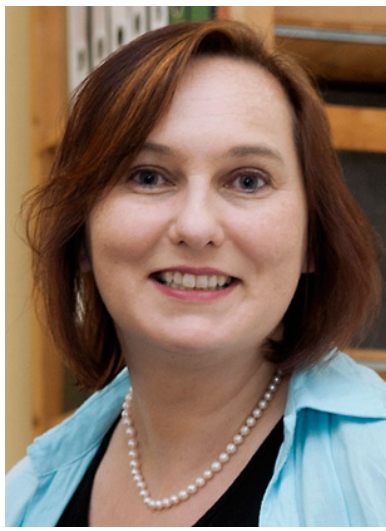

Prof. Dr. Bettina S. Wiese (Diplom in Psychologie an der Universität Marburg; Promotion an der Freien Universität Berlin; Habilitation an der Universität Zürich) hat seit 2011 den Lehrstuhl für Personal- und Organisationspsychologie an der RWTH Aachen inne. Im Zentrum ihrer Forschung steht die Frage, wie Menschen vorgehen, um ihre beruflichen Laufbahnen erfolgreich $\mathrm{zu}$ gestalten und im Alltag eine zufriedenstellende Lebensführung in Arbeits- und Privatleben zu erreichen. 